\title{
Interactions of protons with furan molecules studied by collision-induced emission spectroscopy at the incident energy range of $50-1000 \mathrm{eV}^{\star}$
}

\author{
Tomasz J. Wasowicz ${ }^{1, a}$ and Boguslaw Pranszke ${ }^{2, b}$ \\ 1 Department of Physics of Electronic Phenomena, Gdansk University of Technology, ul. G. Narutowicza 11/12, 80-233 Gdansk, \\ Poland \\ 2 Institute of Experimental Physics, University of Gdansk, ul. Wita Stwosza 59, 80-952 Gdansk, Poland
}

Received 6 May 2016 / Received in final form 5 July 2016

Published online 30 August 2016

(C) The Author(s) 2016. This article is published with open access at Springerlink.com

\begin{abstract}
Investigations of the ion-molecule reactions provide insight into many fields ranging from the stellar wind interaction with interstellar media, up to medicine and industrial applications. Besides the applications, the understanding of these processes is itself a problem of fundamental importance. Thus, interactions of protons with the gas-phase furan molecules have been investigated for the first time in the energy range of 50-1000 eV exploiting collision-induced emission spectroscopy. Recorded spectra reveal emission of the atomic $\mathrm{H}_{\beta}$ to $\mathrm{H}_{\theta}$ lines of the hydrogen Balmer series and the molecular bands of vibrationally and rotationally excited diatomic $\mathrm{CH}$ fragments created in the $\mathrm{A}^{2} \Delta$ and $\mathrm{B}^{2} \Sigma^{-}$electronic states. The measurements of the emission yields of the excited fragments by recording their intensities at different projectile energies have been performed. The highest yields have been observed for production of hydrogen atoms which intensities rapidly decreased with increasing principal quantum number $n$. From the $\mathrm{H}(n=4-7)$ intensity ratios depopulation factors of hydrogen excited states have been determined at each impact energy and possible collisional mechanisms leading to enhanced production of the hydrogen atoms have been suggested. We compare and discuss our results with improved data set of proton collisions with tetrahydrofuran (THF) molecules, the hydrogenated derivatives of furan.
\end{abstract}

\section{Introduction}

It is well-known that apart from photons, stars emit constant stream of charged particles, mainly protons, electrons, $\mathrm{H}_{2}^{+}$and $\mathrm{He}$ ions, a few percent of nuclei with $Z<10$ and very few heavier nuclei $[1,2]$ that can attack nearby medium, i.e planetary and cometary atmospheres, dust grains, meteorites etc. In this viewpoint, studies on resistance and stability of the prebiotic building blocks developed in catalytic reactions on the surfaces of interstellar dust grains against this destructive bombardment are highly required, because they may give knowledge how these species can be formed and preserved in astrophysically relevant environments before being delivered to the terrestrial planets, thus making reliable their contribution to the origin and evolution of life on Earth $[3,4]$. Interactions of protons with biomolecular systems are also of

\footnotetext{
* Contribution to the Topical Issue "Low-Energy Interactions related to Atmospheric and Extreme Conditions", edited by S. Ptasinska, M. Smialek-Telega, A. Milosavljevic, B. Sivaraman.

a e-mail: twasowicz@mif.pg.gda.pl

b Present address: Gdynia Maritime University, ul. Morska 81-87, 81-225 Gdynia, Poland
}

great importance in the studies of radiation damage of the living cells, because, on the one hand, the proton beams are effectively used in hadrontherapy [5], which facilitates $\mathrm{H}^{+}$projectiles to cure cancer, and on the other hand, they are considered for planning future crewed space missions to assess the risk of the exposure of astronauts to the solar wind ejected by the Sun [1]. Furthermore, an increasing number of studies have been devoted to reveal the properties of the ion beam processing and fabrication $[6,7]$.

Furan $\left(\mathrm{C}_{4} \mathrm{H}_{4} \mathrm{O}\right)$ is one of the most fundamental fivemembered heterocyclic aromatic compounds that provides a structural unit of various organic and biologically active substances. For instance, its five-membered ring system consisting of four carbon atoms and one oxygen atom (see Fig. 1) may be regarded to be a building unit in the vitamin B12, biotin [8], conducting polymers [9] and the simple sugars ribose and deoxyribose $(\mathrm{dR})$, the backbone molecules of the RNA and the DNA helix. Moreover, furan and its derivatives play an important role in combustion chemistry, as second-generation biofuels $[10,11]$ or in food and nutrition engineering as a product of thermal degradation of a heat-treated commercial foods $[12,13]$.

Taking into account the relevance of furan molecules in many different fields of astrochemistry, biology and 
(a)

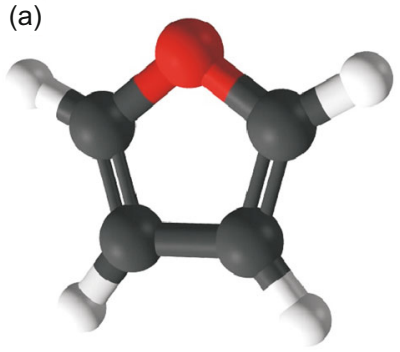

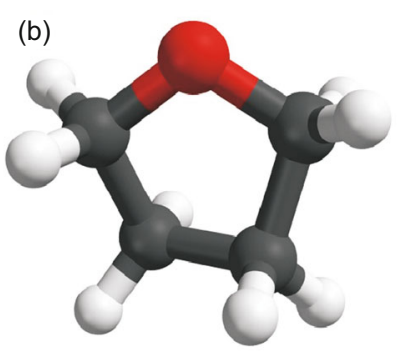

Fig. 1. (a) Furan $\left(\mathrm{C}_{4} \mathrm{H}_{4} \mathrm{O}\right)$ and (b) tetrahydrofuran $\left(\mathrm{C}_{4} \mathrm{H}_{8} \mathrm{O}\right)$ molecules. The colour code: carbon atom is grey, oxygen atom is red, and hydrogen atom is white. industry, they seem to be ideal candidates to characterize the mechanisms of ion-molecule collisions, particularly in the context presented above. Apart from ours conference communication on $\mathrm{He}^{+}$-induced fragmentation of furan molecules [14], to the best of our knowledge, no experimental or theoretical data were reported previously on cationic interactions with either gas- or condensed-phase furan molecules. The present work concentrates on the analysis of the collisional processes occurring in the gasphase furan molecules under protons impact. These mechanisms have been studied using collision-induced emission spectroscopy that allowed identification of the collision products by detecting their emission. Thus, the atomic $\mathrm{H}_{\beta}$ to $\mathrm{H}_{\theta}$ lines of the hydrogen Balmer series and molecular bands of vibrationally and rotationally excited diatomic $\mathrm{CH}$ fragments created in the $\mathrm{A}^{2} \Delta$ and $\mathrm{B}^{2} \Sigma^{-}$electronic states have been recognized in the recorded spectra. The energetic dependences of the intensities of the excited fragments i.e. the emission yields have been recorded. For comparison, the emission yields in the $\mathrm{H}^{+}+$tetrahydrofuran collisions have been also remeasured in the $20-1000 \mathrm{eV}$ energy range, but with smaller energy step size than in our previous studies [15]. For both impact systems the highest yields have been observed for production of the excited $\mathrm{H}$ atoms which intensities rapidly decrease with increasing principal quantum number $n$. Moreover, the intensity ratios of the $\mathrm{H}(n=4)$ emissions and the $\mathrm{A}^{2} \Delta \rightarrow \mathrm{X}^{2} \Pi_{r}$ bands of the $\mathrm{CH}$ show that the $\mathrm{H}(n=4)$ are more abundant fragments in the entire studied energy range. These observations give clear indication that collision processes are dominated by an electron transfer from the target molecules to projectiles leading to enhanced production of the hydrogen atoms arising mostly from neutralization of protons and excited to lower lying states. Depopulation studies of higher excited states of hydrogen also support this picture.

\section{Experiment}

The experiment was carried out at the University of Gdansk exploiting the collision-induced emission spectroscopy developed in Göttingen by Ottinger [16]. A detailed technical information was described in references $[16,17]$. The sketch of the apparatus and methodology of collecting of the data was presented in our previous papers $[15,18]$. Briefly, the applied set up allowed measurement and identification of the reaction products by detecting their emission and it consisted of a water cooled cation source, magnetic mass selector, collision cell, and an optical spectrometer equipped with sensitive multichannel photon-counting detector [15-18]. The source was of a colutron-type and it produced protons from $\mathrm{H}_{2}$ gas being under pressure of $100 \mathrm{~Pa}$. The distance between cathode and anode in the cation source was set at $5 \mathrm{~mm}$. An anode-to-cathode voltage was maintained at $100 \mathrm{~V}$ and a discharge current was kept at $500 \mathrm{~mA}$. To get the ions of desired parameters the steel mesh was mounted between anode and cathode. This allowed enhancing production of ions and better focusing of the plasma on the hole in anode. Additionally the extracting aperture had a characteristic shape of the sombrero hat. Protons were extracted from the discharge region by $1000 \mathrm{~V}$ voltage and were directed via a $1 \mathrm{~mm}$ dia. hole in the anode into the $60^{\circ}$ magnetic mass selector, where they were mass selected by an electromagnet. Leaving this chamber the cations were slowed down to the desired energy by three immersion lenses. Then the $\mathrm{H}^{+}$beam was shaped by a slit $1 \mathrm{~mm}$ wide and $10 \mathrm{~mm}$ high positioned at the end of the immersion lenses system, $12 \mathrm{~mm}$ before the collision chamber and reached the collision chamber. The ion beam collided with the target gas in a grounded collision cell $24 \mathrm{~mm}$ in length. Only the central section of the collision cell, $12 \mathrm{~mm}$ long, represented the effective observation region that was viewed at $90^{\circ}$ to proton beam axis by the optical spectrometer. Passing through the collision gas, the beam is attenuated, by between a few percent to about $50 \%$ [16]. But by adjusting the geometrical path of the beam this effect was to some extent reduced. The proton beam current was measured on the rear slit of the collision chamber. Typical proton beam current in the interaction region was about $1.2 \mathrm{nA}$ at $50 \mathrm{eV}$ and $\sim 10 \mathrm{nA}$ at $1000 \mathrm{eV}$. Next, the proton beam passed through target molecule vapours, which were provided into the collision cell through a gas inlet system. The furan sample was purchased from Sigma Aldrich, Poland, with a declared purity of $>99 \%$. Furan is liquid at the room temperature, but it is very volatile and can be measured without heating the sample, which was only outgassed through freeze-pump-thaw cycles, until no release of contaminating gases from the melting ices was observed. It was found that the emission signal is linear function of target gas pressure up to 30 mTorr. Therefore, in order to avoid any secondary collision mechanisms the pressure of vapours was maintained at 15 mTorr, as determined with the Barocel capacitance manometer. Light emitted from the interaction region was reflected by a concave $\mathrm{Al}$ mirror and focused onto the entrance slit of a McPherson 218 spectrograph equipped with two gratings: the $300 \mathrm{~L} / \mathrm{mm}$ blazed at $500 \mathrm{~nm}$ or $1200 \mathrm{~L} / \mathrm{mm}$ 
blazed at $250 \mathrm{~nm}$. It was then detected by a 1024 channel "Mepsicron" detector, sensitive in the 180-600 nm wavelength range. In the experiment we used both gratings. In order to obtain high resolution spectra the grating having $1200-\mathrm{L} / \mathrm{mm}$ was used. This allowed an accurate identification of the spectral components. The spectrograph operated with the $300-\mathrm{L} / \mathrm{mm}$ grating in order to measure the emission yields. These yields were obtained by integrating over the lines and bands areas containing the vibrational and rotational lines. The background signal was measured by cutting off the furan beam flow and it was subtracted from the original spectra. Then, the spectra were corrected for the wavelength dependence of the sensitivity of the optical detection channel. The resulting emission yields were next normalized to the cation beam current ( $\mathrm{nA})$ and recording time (1 min).

The polarization effects $[19,20]$ may influence the intensities obtained with the present setup. Such effects are of particular relevance for the determination of the absolute values of the cross sections. In the case of relative cross sections they seem to be less important. Nevertheless a polarization correction is required. Van Zyl et al. [21-23] have measured the polarization of $\mathrm{H}(n=$ $3,4 \rightarrow 2$ ) radiations in a collisions of $\mathrm{H}^{+}$and $\mathrm{H}$ with noble gases. The polarizations of these lines vary between 5 and $30^{\circ}$ at the $30-1000 \mathrm{eV}$ energy range. According to Moiseiwitsch and Smith [19] a real intensity $I$ is given by $I=I\left(90^{\circ}\right)(1-P / 300)$, where $I\left(90^{\circ}\right)$ is apparent intensity and $P$ is polarization of radiation. Thus, the polarizations of $5-30 \%$ induce errors between $2-9 \%$. In addition, Hoekstra et al. [24] have carefully analyzed the possible contributions to polarization effects for the He $(n=4 \rightarrow 3)$ transition. They showed that anisotropy of radiation and instrumental effects can to some extend cancel out and the $5 \%$ error was estimated due to neglecting polarization effects [24]. Nevertheless, an instrument that is suitable for the measurement of angle-integrated intensities of excited fragments could be constructed by installing a present detection system at the so-called magic angle $\left(54.7^{\circ}\right)$ with the ion beam axis and tilting the spectrometer $45^{\circ}[19,20,24]$. Alternatively, a setup of two spectrometers mounted at $0^{\circ}$ and $90^{\circ}$ could be used, which would allow the determination of the angular asymmetry parameters of emitted radiation.

\section{Results and discussion}

\subsection{Identification of fragments}

High resolution emission spectra measured for the collisions between the $\mathrm{H}^{+}+\mathrm{THF}$ and $\mathrm{H}^{+}+$furan at the energy of $1000 \mathrm{eV}$ are displayed in Figure 2. Both spectra reveal the hydrogen lines of the Balmer series, $\mathrm{H}_{\beta}$ to $\mathrm{H}_{\theta}$, due to the excited hydrogen $\mathrm{H}(n)$ atoms, $n=4-9$ and point also at production of the excited $\mathrm{CH}$ diatomic molecules which were identified by their decay with emission of the molecular systems of $\mathrm{CH}\left(\mathrm{A}^{2} \Delta \rightarrow \mathrm{X}^{2} \Pi_{r}, \mathrm{~B}^{2} \Sigma^{+} \rightarrow \mathrm{X}^{2} \Pi_{r}\right)$.

For the $\mathrm{H}^{+}+\mathrm{THF}$ collisions the $\mathrm{CH}$ bands are barely distinguishable, but for furan we observe stronger emission. In contrary, luminescence spectrum measured for the

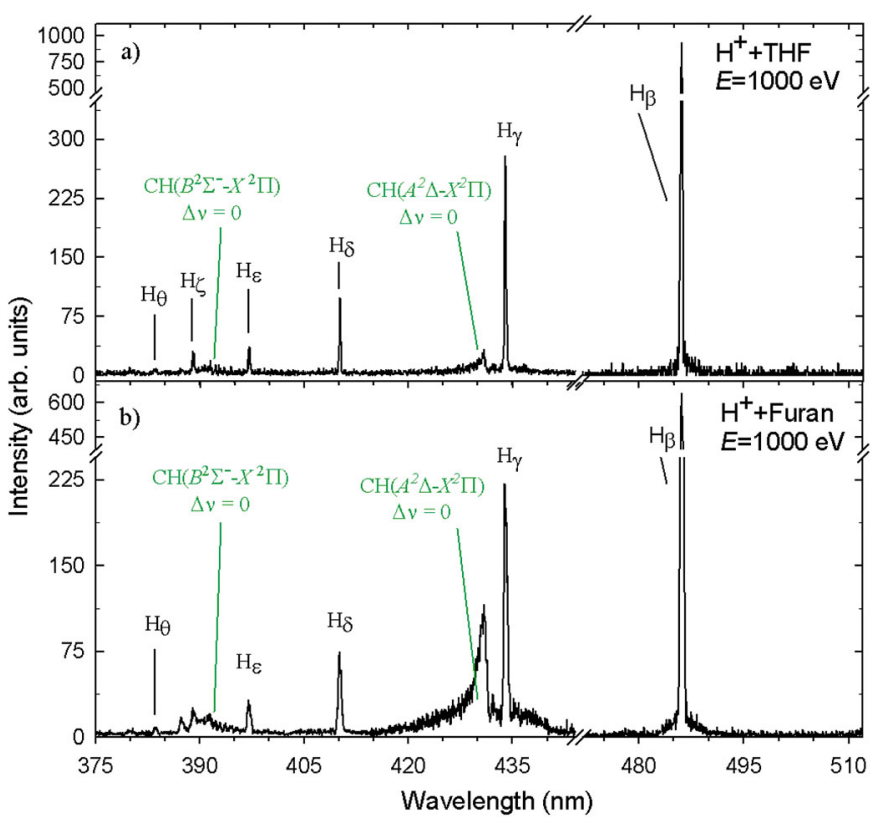

Fig. 2. Emission spectra measured with an optical resolution $\Delta \lambda$ of $0.4 \mathrm{~nm}$ (FWHM) for collisions of the $\mathrm{H}^{+}$cations with (a) tetrahydrofuran and (b) furan. The spectra were not corrected for the wavelength dependence of the sensitivity of the detection system. The data for $\mathrm{H}^{+}+\mathrm{THF}$ collisions was taken from [15].

$\mathrm{He}^{+}$-induced fragmentation of furan molecules not only reveals much stronger emission of $\mathrm{CH}$ radical, but also shows emission of the $\mathrm{C}_{2}$ molecule and the $\mathrm{C}$ and He atoms [14]. Also fluorescence spectrum measured in the studies of electron impact excitation of furan [25] presents pronounced intensity of the diatomic $\mathrm{CH}$ fragments formed in the $\mathrm{A}^{2} \Delta, \mathrm{B}^{2} \Sigma^{-}$and even $\mathrm{C}^{2} \Sigma^{-}$electronic states and the $\mathrm{C}_{2}$ fragments in the $\mathrm{d}^{3} \Pi_{g}$ excited state. Present results of fragmentation of furan molecule may also be compared to the photon [26-28], cation [15] and electron $[29,30]$ impact fragmentation of other five- and six-membered heterocyclic molecules. Again in contrast to present measurement all these studies demonstrated major fragmentation of the target molecules into several different atomic and diatomic fragments. Hence, presented comparison indicates that collisions of protons with furan (and also THF) would not lead to efficient dissociation of the target molecules.

It can be noticed that $\mathrm{CH}$ molecule is excited vibrationally and rotationally. Therefore, we have calculated the synthetic $\mathrm{CH}$ spectrum utilizing the LIFBASE molecular spectra simulation program [31] with the use of vibrational and rotational constants of the $\mathrm{A}^{2} \Delta, \mathrm{B}^{2} \Sigma^{+}$ and $\mathrm{X}^{2} \Pi_{r}$ electronic states of $\mathrm{CH}$ from [32-37]. Similar computer procedure, employed in the analysis of spectra of diatomic molecules, occurring in cation- and photoninduced fragmentation of five- and six-membered heterocycles, has been recently proven to be efficient and dependable $[15,18,27,28,38]$. Calculations show that in the collisions of furan with the $\mathrm{H}^{+}$(Fig. 3a) the experimental $\mathrm{CH}\left(\mathrm{A}^{2} \Delta \rightarrow \mathrm{X}^{2} \Pi_{r}\right)$ emission spectrum was reproduced well for the vibrational and rotational populations, assumed to be Boltzmann, governed by the characteristic 


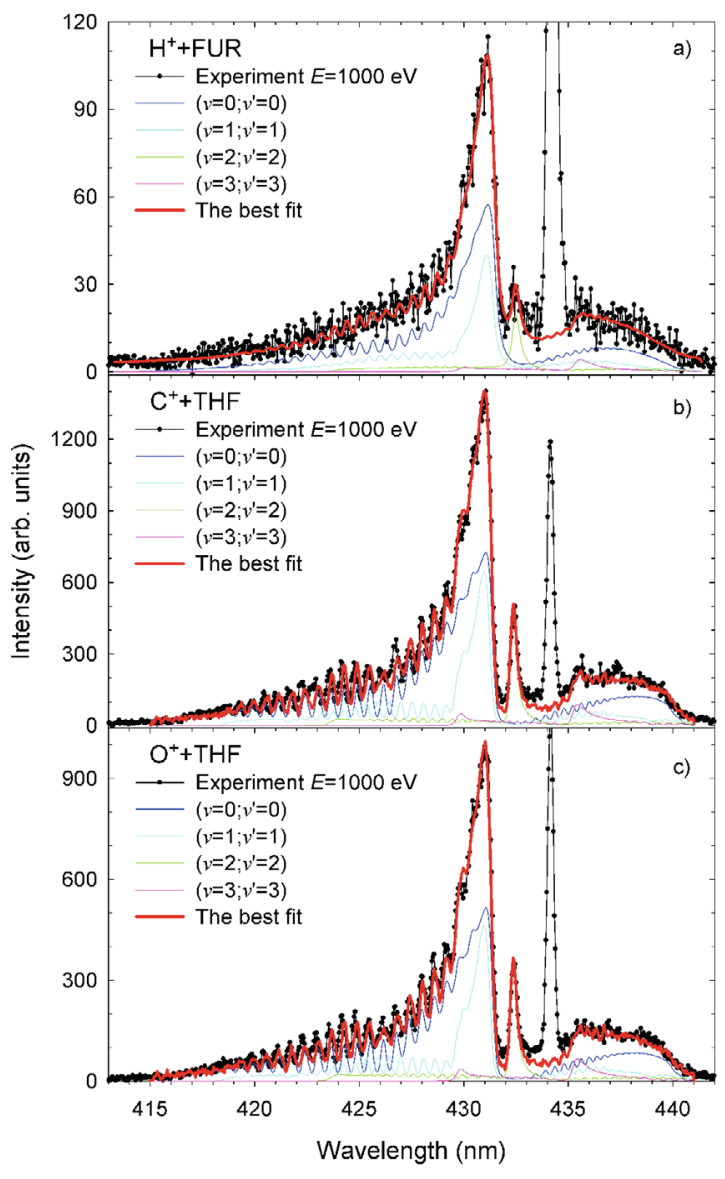

Fig. 3. The simulated (red contours) and experimental (black dots) $\mathrm{CH}\left(\mathrm{A}^{2} \Delta \rightarrow \mathrm{X}^{2} \Pi_{r}\right)$ spectra obtained in the (a) $\mathrm{H}^{+}+$furan, (b) $\mathrm{C}^{+}+\mathrm{THF}$, (c) $\mathrm{O}^{+}+\mathrm{THF}$ collisions. The data for $\mathrm{C}^{+} / \mathrm{O}^{+}+\mathrm{THF}$ collisions was taken from [15].

temperatures of $T_{v}=7100 \mathrm{~K}$ and $T_{r}=3200 \mathrm{~K}$, respectively. In the simulation we have used the Voigt profile for the apparatus function with a resolution $\Delta \lambda$ of $0.39 \mathrm{~nm}$ (FWHM). In Figures $3 \mathrm{~b}$ and $3 \mathrm{c}$ we have also shown fittings performed for the collisions between the $\mathrm{C}^{+}+\mathrm{THF}$ and $\mathrm{O}^{+}+\mathrm{THF}$, respectively. For those impact systems, the best agreement between simulated and measured spectra was achieved using the vibrational and rotational temperatures of $T_{v}=5200 \mathrm{~K}, T_{r}=8900 \mathrm{~K}$ (Fig. 3b) and $T_{v}=5000 \mathrm{~K}, T_{r}=9000 \mathrm{~K}$ (Fig. 3c), respectively [15]. Here, we have also used the Boltzmann distributions of populations and the Voigt profile for the apparatus function, however with a resolution $\Delta \lambda$ of $0.35 \mathrm{~nm}$ (FWHM). The collisions with protons yield a vibrationally hotter, but rotationally cooler $\mathrm{CH}$ product than interactions with the $\mathrm{C}^{+}$and $\mathrm{O}^{+}$cations. This indicates that during those collisions the $\mathrm{CH}$ fragments were produced in different physical conditions. This may be related to the fact that protons do not induce effective fragmentation in contrast to $\mathrm{C}^{+}$and $\mathrm{O}^{+}$cations.

\subsection{Emission yields}

In Figure 4 we present the emission yields of the $\mathrm{H}(n=4)$ (upper panel) and $\mathrm{CH}\left(\mathrm{A}^{2} \Delta\right)$ (lower panel) frag-

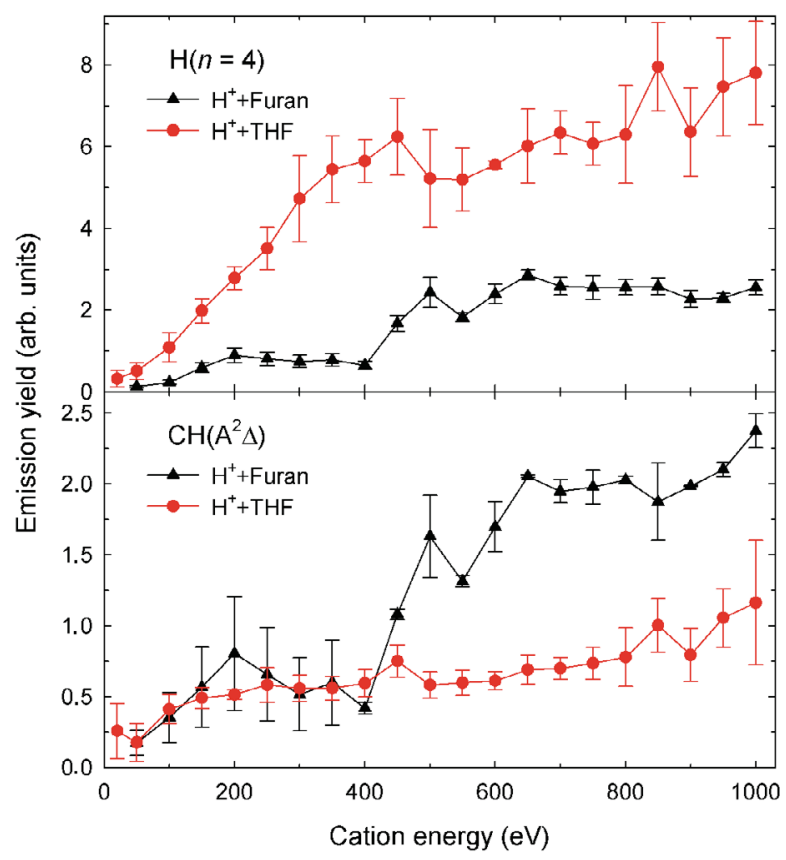

Fig. 4. The emission yields of the excited $\mathrm{H}(n=4)$ and $\mathrm{CH}\left(\mathrm{A}^{2} \Delta\right)$ fragments obtained in collisions of $\mathrm{H}^{+}$with furan and tetrahydrofuran. The experimental uncertainties of the emission yields are the mean standard deviations obtained from several independent measurements performed at fixed proton energies. Due to the ion beam energy spread and thermal motion of the target, the uncertainty of collision energy was estimated to be $3 \mathrm{eV}[39]$ and it is smaller than the size of symbols.

ments measured in collisions of furan and tetrahydrofuran molecules with the $\mathrm{H}^{+}$cations plotted as function of the energy of the projectiles. It is seen that these functions have different, structurized shapes.

For instance, the $\mathrm{H}(n=4)$ curve obtained in collisions of $\mathrm{H}^{+}$with furan increases with energy and reaches the first maximum at $200 \mathrm{eV}$. Above $400 \mathrm{eV}$ it rises rapidly to maximum at $650 \mathrm{eV}$ and above $650 \mathrm{eV}$ it reaches plateau. The $\mathrm{CH}\left(\mathrm{A}^{2} \Delta\right)$ emission yield resembles the $\mathrm{H}(n=4)$ curve up to $800 \mathrm{eV}$, but in contrast above $850 \mathrm{eV}$ it starts rising. The measurements for the $\mathrm{H}^{+}+\mathrm{THF}$ collisions were performed for the $20-1000 \mathrm{eV}$ projectiles energies, which covered the velocity range of $62-439 \mathrm{~km} / \mathrm{s}$. The $\mathrm{H}(n=4)$ and $\mathrm{CH}\left(\mathrm{A}^{2} \Delta\right)$ energy dependences measured in this collision system are slightly different from former yields. The $\mathrm{H}(n=4)$ curve increases rather rapidly above $20 \mathrm{eV}$ up to a maximum at $450 \mathrm{eV}$. Above $500 \mathrm{eV}$ it rises gradually and does not show resonances in this energy range. On the other hand, the $\mathrm{CH}\left(\mathrm{A}^{2} \Delta\right)$ emission yield rises very slowly with increasing energy. Other yields (not presented here) resemble to some extent curves shown in Figure 4. Moreover, for both impact systems, the intensities of $\mathrm{H}(n)$ rapidly decrease with increasing principal quantum number $n$.

\subsection{Collisional processes}

Reaction channels generating the observed fragments may be triggered by four collision processes (see [15] and 


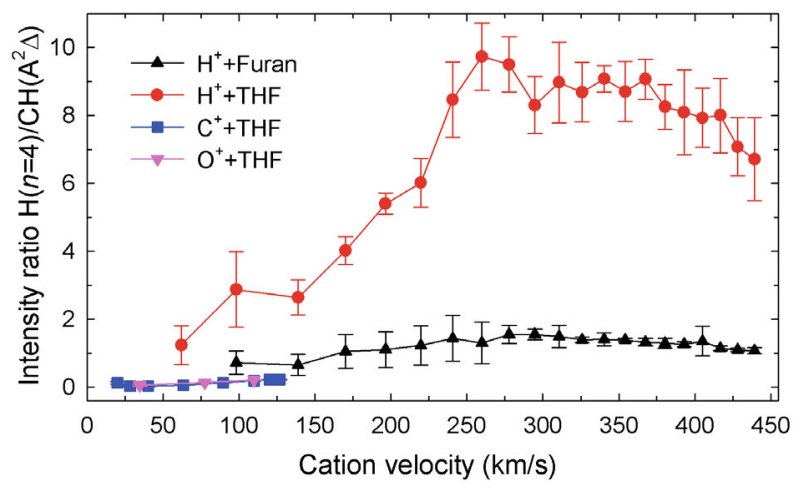

Fig. 5. The ratio of fragmentation yields of the $\mathrm{H}(n=4)$ to $\mathrm{CH}\left(\mathrm{A}^{2} \Delta\right)$ fragments in the collisions of $\mathrm{H}^{+}$with furan as well as $\mathrm{H}^{+}, \mathrm{C}^{+}$, and $\mathrm{O}^{+}$with THF. Results for the collisions of $\mathrm{O}^{+}$ and $\mathrm{C}^{+}$with $\mathrm{THF}$ were taken from reference [15].

references therein). The first process is an electron capture from the target molecule to the projectile followed by fragmentation of molecule's cation. This process takes place at large projectile-target distance. The second process is dissociative ionization this is direct ionization of the target molecule and further fragmentation of molecule's cation. The third one starts from excitation of target molecule and further fragmentation of the excited molecule (dissociative excitation). Both dissociative processes require closer collisions regime. The last mechanism involves the transient cation-molecule complex formation prior to fragmentation. Because, formation of the ion-molecule complexes may be the effect of the ion-dipole interaction, it may occur at relatively large projectile-target distances.

It is demanding to establish unambiguously in such kinds of experiments which collisional mechanism plays a major role in yielding the observed fragments. However, our results may comprise some information about them. Indeed, from the emission spectra shown in Figure 2 and the obtained values of the emission yields displayed in Figure 4 it is discernible that the $\mathrm{H}(n)$ are the most abundant fragments in the entire studied energy range, while the $\mathrm{CH}$ radicals have lower intensities. This can be clearly seen if we calculate the intensity ratios of the observed fragments as quotients of their fragmentation yields. An evolution of the $\mathrm{H}(n=4)$ to $\mathrm{CH}\left(\mathrm{A}^{2} \Delta\right)$ ratio in the studied collision systems is illustrated in Figure 5. The experimental results determined in the collisions of $\mathrm{O}^{+}$and $\mathrm{C}^{+}$with THF [15] are also given in Figure 5 for comparison. In all collisional systems the $\mathrm{CH}$ fragments arise due to disintegration of the molecular ring. Moreover, in the collisions of $\mathrm{O}^{+}$and $\mathrm{C}^{+}$with THF the hydrogen atoms must be detached from the molecular structure of THF or furan. On the contrary, in proton impact the excited hydrogen fragments may come from both neutralized projectiles and/or fragmentation of target molecule. Thus, the $\mathrm{H}(n=4) / \mathrm{CH}\left(\mathrm{A}^{2} \Delta\right)$ ratio characterizes the magnitude of the collisional mechanisms. It is visible from Figure 5 that values of this ratio obtained for protons are much higher than that for the $\mathrm{C}^{+}$ and $\mathrm{O}^{+}$cations. The enhancement of hydrogen peaks in the present experiment cannot be explained by fragmen- tation of the target molecule itself, because on one hand the formation of the $\mathrm{CH}$ radicals is minor as compared to other impact systems $[14,15,25-30]$ and on the other hand the production of the excited hydrogen atoms from neutralized protons, due to electron capture mechanism, is energetically the most favored channel [15].

Moreover, the ratios in proton impact with both molecules firstly rise to $260 \mathrm{~km} / \mathrm{s}(350 \mathrm{eV})$, and they stabilise up to $370 \mathrm{~km} / \mathrm{s}(\sim 700 \mathrm{eV})$, then start decreasing. These observations can be elucidated as follows. The ab initio calculations of the collisions of $\mathrm{C}^{q+}(q=2-4)$ carbon cations and protons with deoxyribose $[40,41]$ show sharp avoided crossings between the entrance channel $\mathrm{H}^{+}+\mathrm{dR}$ and the different $\mathrm{H}+\mathrm{dR}^{+}$electron transfer levels at the potential energy curves at large distance of $R=2.5 \AA$. These avoided crossings induce efficient electron charge transfer because this process is driven by the nonadiabatic interactions between the adiabatic potential curves at their points of closest approach, but they would decrease the probability of dissociation $[40,41]$. Our results suggest that similar conditions may ensue for the $\mathrm{H}^{+}+\mathrm{THF}$ and $\mathrm{H}^{+}+$furan collisions. The ratio of emission yields firstly increases and then becomes almost constant, because at higher energies the electron capture would be a dominant mechanism, while at lower energies apart from the charge transfer reaction, the complexation, dissociative excitation and ionization processes may simultaneously occur, but with low cross sections. This discussion can also be conclusive for interpretation of the shapes of the emission yields.

It is of note that electron charge transfer can occur even in the systems where the resonant electron capture is energetically forbidden. Indeed, we have recently observed signatures of single and double electron capture reactions in the $\mathrm{He}^{+}$and $\mathrm{He}^{++}$collisions with pyridine [42]. These reactions occurred even though the resonant electron capture from the highest occupied molecular orbital (HOMO) of pyridine to the $\mathrm{He}^{+}$was energetically precluded because of incongruity between the energy levels of these reactants.

It is well-known that furan is planar molecule (see Fig. 1), but THF has spatial structure (Fig. 1) and it exists in two puckered conformational forms [43]. In consequence its charge density occupies larger volume in every direction than that of furan molecule. Thus, protons can easier interact with THF molecules enhancing the probability of charge transfer which is highly anisotropic [44]. Moreover, the $\mathrm{H}$ atoms may be also produced throughout fragmentation of target molecule. In contrast to furan, the THF molecule possesses more hydrogens that can be detached from the ring and excited due to the dissociative excitation or ionization processes. For all those reasons, the $\mathrm{H} / \mathrm{CH}$ ratio is likely higher for proton-THF collisions than for proton-furan ones (see Fig. 5).

\subsection{Major fragmentation channels}

As it was argued in the preceding section, the highest yields obtained for production of the excited hydrogen atoms in the $\mathrm{H}^{+}+$furan collisions indicate that fragmentation processes occur with small cross sections and are 
mostly preceded by the electron transfer from the target molecules to protons. It is thus expected that in the $\mathrm{H}^{+}+$furan collisions possible reaction channels proceed through fragmentation of the parent cation of furan $\left(\mathrm{C}_{4} \mathrm{H}_{4} \mathrm{O}^{+}\right)$. Because in fragmentation of polyatomic molecules a double sequential dissociation reaction is plausible, where one of the primary dissociation products undergo further decomposition [28], the $\mathrm{C}_{4} \mathrm{H}_{4} \mathrm{O}^{+}$may dissociate into charged fragments and corresponding neutral species. The charged fragments can easily be measured and identified by the mass spectrometric techniques. If the mass spectrum reveals high intensity of particular cation one may assume that corresponding neutral counterpart also is effectively produced. This may indicate that smaller fragments (i.e. the atoms or diatomic molecules) may come from subsequent decomposition of those abundant neutral dissociation products. Thus, the species observed in the present experiment ought to correlate with the ionic fragments observed in the furan mass spectra. Because of the limited number of ion-molecule reactions that have been studied involving furan molecules, no direct comparisons could be made to literature data. However, there was a study that provided electron impact fragmentation data for furan [45] that can be directly compared with our product distribution. In this context, the simplest fragmentation process is expected in the production of atomic hydrogen $\mathrm{H}(n)$ fragments. These may directly result from the abstraction of $\mathrm{H}$ atoms from the furan parent ion ring. But the mass spectrometric studies showed unexpectedly low intensity of the $\mathrm{C}_{4} \mathrm{H}_{3} \mathrm{O}^{+}$cation [45]. Therefore, direct detachment of neutral $\mathrm{H}(n)$ from furan parent ion has low probability and fragmentation processes must occur via the ring opening by scission of the weakest $\mathrm{C}-\mathrm{O}$ bond and further rupture of the $\mathrm{C}-\mathrm{H}$ and/or $\mathrm{C}-\mathrm{C}$ bonds. The most abundant fragment in the mass spectrum of the cations of furan was identified as $\mathrm{C}_{3} \mathrm{H}_{3}^{+}(\mathrm{m} / q=39)[45]$ which corroborates the above scenario. The corresponding neutral fragment is then the $\mathrm{HCO}$ molecule, which may further decompose into neutral $\mathrm{H}$ and $\mathrm{CH}$ fragments. Other abundant cation was observed at mass $29 \mathrm{amu}$, and it corresponds to $\mathrm{HCO}^{+}$. The neutral moiety is then the $\mathrm{C}_{3} \mathrm{H}_{3}$ which may also undergo fragmentation yielding the observed fragments.

\subsection{Populations of $H(n)$}

Now we are focusing our attention on the formation of the $\mathrm{H}(n)$ atoms, because the intensity ratios of Balmer lines, namely the Balmer decrements, have several applications. For instance, they are used to estimate interstellar dust extinction $[46,47]$, to diagnostics of high-power plasmas [48], and to investigate the interaction of plasmas with surfaces and also of gas flows with near-surface plasmas [49]. The values of the Balmer decrements are set by quantum mechanics, i.e. the line emission intensities $I$ for transitions occurring from the $n l$ upper states onto $n^{\prime} l^{\prime}$ lower states are inversely proportional to the third power of principal quantum number $n$ [50]: $I \sim n^{-3}$. This dependence was expected for excitation of atoms and for molecules in high

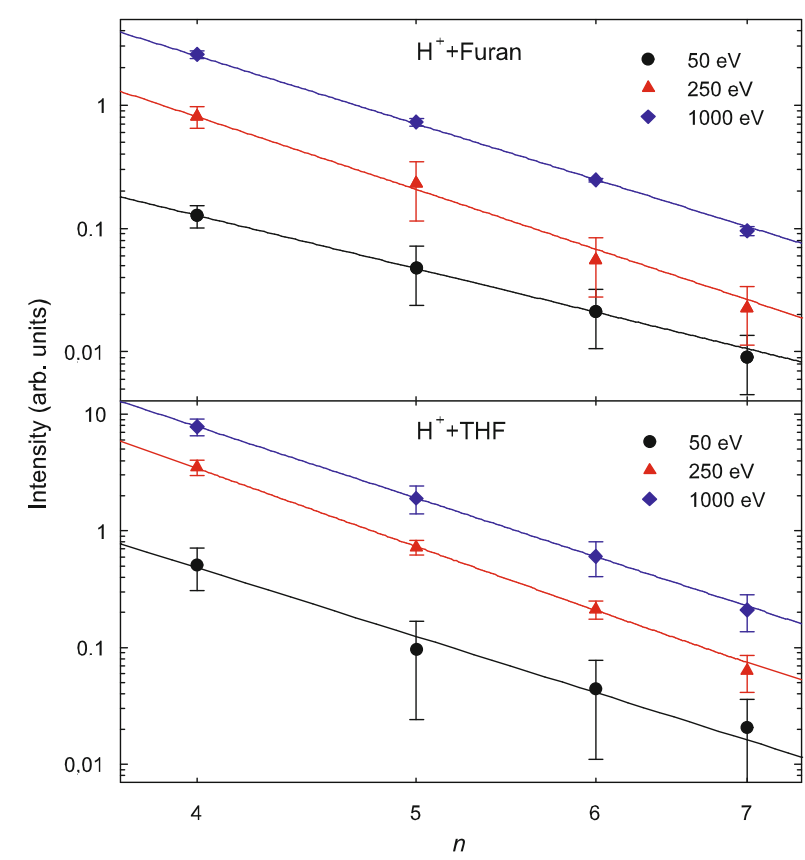

Fig. 6. The emission intensity of the lines of the Balmer series as a function of the principal quantum number $n$. The solid lines show the best fits to the experimental points (see details in the text).

Rydberg states. Thus, any deviation from the expected value may give an information on collisional mechanisms. If this parameter is close to -3 it may be taken as an indication of emission by hydrogen stemming from dissociation of the target molecule. The lower values may suggest that emission comes from excited projectiles due to the charge transfer processes and can be understood as a measure of depopulation of the hydrogen excited states. Thus, for each impact energy, the intensities of the Balmer lines (integrated over the band shapes) were plotted as a function of the principal quantum number $n$ of the excited $\mathrm{H}(n)$ atoms. Figure 6 displays example diagrams for the selected energies delineated in the log-log plot.

As is seen in Figure 6, the intensities of Balmer lines are linear functions of $n$ and thus they can be approximated by an $n^{K}$ expression, where $K$ is depopulation factor. According to this formula, at each impact energy the straight line was fitted to the experimental points using a weighted regression method with the weights proportional to the experimental errors. Example fits are presented in Figure 6 as the solid lines. As consequence, the slopes of the regression lines give the $K$ values which plotted as a function of the projectiles energy are presented in Figure 7.

It is clearly seen in Figure 7 that for collisions with protons the values of the $K$ constants depend on the collision energies and show major depopulation of higher lying states of hydrogen. In fact, in the entire energy range depopulation factors are lower than -3 . In particular, between the $20-200 \mathrm{eV}$ the $K$ values rapidly decrease from -4.4 to -6.2 and -4.8 to -7.3 for $\mathrm{H}^{+}+$furan and $\mathrm{H}^{+}+\mathrm{THF}$ collisions, respectively. Then above $200 \mathrm{eV}$, 


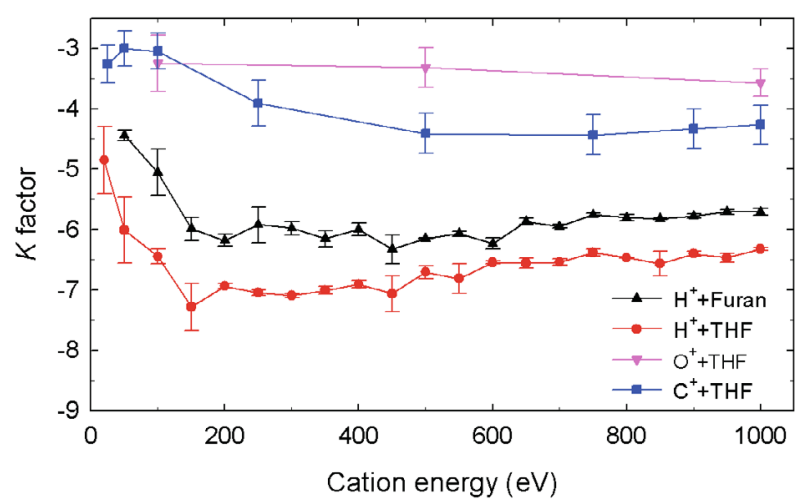

Fig. 7. Energetic dependences of the depopulation factors.

in both impact systems, the $K$ factors very slowly raise with increasing of the $\mathrm{H}^{+}$projectiles energies, but even that the maximum values are very low and are equal to -5.7 and -6.3 for furan and THF, respectively. The present energetic dependences of the depopulation factors may be compared with that obtained from fragmentation of tetrahydrofuran molecules into the $\mathrm{H}(n)$ atoms induced by the $\mathrm{O}^{+}$and $\mathrm{C}^{+}$impact [15]. Thus, we have determined the $K$ factors from the Balmer decrements measured in THF and in Figure 7 they are also presented for comparison. For the energies below $100 \mathrm{eV}$ in the $\mathrm{C}^{+}$collisions with THF the depopulation factors were found to be -3.0 and they decrease to -4.4 with the increase of the $\mathrm{C}^{+}$ cations energies. In contrast to those results, the values of the $K$ factors obtained in the $\mathrm{O}^{+}$impact, on average in the presented energy range, are equal to -3.3 .

In general, the differences in the $K$ constants may point to changes in the mechanisms, which populate the $\mathrm{H}(n)$ atoms in the dissociation of furan and tetrahydrofuran molecules. As it was already mentioned in the text, at low energies/velocities the close collisions of the cations with the target molecules dominate, and the complexation, dissociative excitation and ionization processes may occur simultaneously. This leads to enhancement of the probability of population of higher excited states of the $\mathrm{H}(n)$ atoms, because such hydrogens may be produced via several different channels. In consequence, the values of the $K$ factors should be close to -3.0 . This is exactly what we observe in the $\mathrm{O}^{+}$and $\mathrm{C}^{+}$collisions (see Fig. 6). For collisions with protons the situation is slightly different, because protons induce all these processes, however with very low cross sections. Lower probability of population of higher lying states thereby reduces the values of the $K$ factors. That is why for protons major depopulation of hydrogen's higher excited states was observed even at lower energies. As the impact energies rise the cations become fast and time of interaction with the target molecule is very short. Although the distance between reactants becomes larger, the projectiles still may feel the energy potential of the target molecule. Thus, the only one mechanism (to be precise the electron capture) starts governing over other collisional processes and higher excited states of the $\mathrm{H}(n)$ atoms are not effectively populated. Therefore, the $K$ factors decrease at higher velocities and reach their minimal values. It is of note that collisional excitation of the electronic system of the target molecule, this is the electronic stopping [51], is more extensive for the $\mathrm{C}^{+}$cations than that for $\mathrm{H}^{+}[15]$. Hence, the depopulation in the $\mathrm{C}^{+}+\mathrm{THF}$ collisions exists, nonetheless it is not so efficient. In contrast, impact with oxygen cations prompts production of the $\mathrm{H}(n)$ atoms that have the most effectively populated of higher excited states. This observation may be explained as an effect of the reduction of the electron transfer events. Indeed, in our previous studies [15] we could not identify any signatures of the electron capture mechanism in the $\mathrm{O}^{+}+\mathrm{THF}$ collisions. Since the $\mathrm{O}^{+}$cations are rather slow $(1000 \mathrm{eV}$ energy corresponds to velocity of $110 \mathrm{~km} / \mathrm{s}$ ) the close collisions regime was most likely significant in the $\mathrm{O}^{+}+\mathrm{THF}$ collisions [15]. Thus, the complexation, dissociative excitation and ionization could occur at the same time leading to major fragmentation of THF and production of the hydrogen atoms in the excited states that are populated according to $n^{-3}$.

It is clearly seen that depopulation is prevalent during present charge transfer reactions, but the question is why? It is well-known that branching ratios and lifetimes of certain states may be modified by electric fields via Stark mixing. Not only extremely high electric fields [52-55] can affect the system, but also the residual electric field present in the chamber can influence on the wave functions of the excited states [24]. The states having the same value of the total angular momentum quantum number $j$ are the most prone to such mixing [50]. For example, for the $s_{1 / 2}$ and $p_{1 / 2}$ levels at $n=2,3,4$ and 6 the critical field strengths, i.e. the minimum fields which will cause full mixing, are relatively high 475, 58, 12 and $1.7 \mathrm{~V} / \mathrm{cm}$, respectively [50]. But for the $p_{3 / 2}$ and $d_{3 / 2}$ levels the critical fields are much lower and are equal to 2 and $\frac{1}{2} \mathrm{~V} / \mathrm{cm}$ at $n=3$ and $n=4$, respectively [50]. Present setup was built to study very weak chemiluminescent reactions occurring mainly at lower energies. To reduce the unwanted effects each part of the setup was constructed in separate, shielded chamber. Thus, we only expect the electric fields due to the space charge of the proton beam and produced by the movement of projectiles in the earth's magnetic field. In the simple estimation [24] the first contribution leads to the field strengths of an order of nano $\mathrm{V} / \mathrm{cm}$ and can be neglected. The earth's magnetic field in the Gdansk region is on average equal $50 \mu \mathrm{T}\left(B_{\text {vertical }}=-47 \mu \mathrm{T}\right.$; $B_{\text {horizontal }}=17 \mu \mathrm{T}$, see [56]). Taking into account present velocity limits, such a magnetic field may not produce the electric field strengths higher than $0.22 \mathrm{~V} / \mathrm{cm}$. This value is small enough to neglect the Stark mixing effects for $n=3,4$. However the critical field strength decreases very rapidly as $n^{-5}$ [50] and the Stark mixing may contribute in depopulation of the $n=6,7,8, \ldots$ levels. But now the question is why do not we observe such an effect in collisions with $\mathrm{C}^{+}$and $\mathrm{O}^{+}$? The answer is very simple: the most probable velocity in a molecular beam is of an order $300 \mathrm{~m} / \mathrm{s}$ [57] (due to conservation of momentum the hydrogen fragment velocity may be even lower) which is too low to generate the electric fields capable of mixing of the excited states. 


\section{Summary}

In the present work the interactions of protons with the gas-phase furan molecules have been investigated for the first time in the energy range of 50-1000 eV. For comparison, the $\mathrm{H}^{+}+$tetrahydrofuran collisions have been once again studied in the 20-1000 eV energy range, but with smaller energy step size than in our previous studies [15]. Collision-induced emission spectroscopy was applied to detect the atomic lines of the hydrogen Balmer series and molecular bands of vibrationally and rotationally excited diatomic $\mathrm{CH}$ radicals by recording the $\mathrm{A}^{2} \Delta \rightarrow \mathrm{X}^{2} \Pi_{r}$ and $\mathrm{B}^{2} \Sigma^{-} \rightarrow \mathrm{X}^{2} \Pi_{r}$ emission bands. For both impact systems the highest yields have been observed for production of the excited $\mathrm{H}$ atoms which intensities rapidly decrease with increasing principal quantum number $n$. Moreover, the intensity ratios of the $\mathrm{H}(n=4)$ emissions and the $\mathrm{A}^{2} \Delta \rightarrow \mathrm{X}^{2} \Pi_{r}$ bands of the $\mathrm{CH}$ show that the $\mathrm{H}(n=4)$ are more abundant fragments in the entire studied energy range. These observations suggest that the excited hydrogen atoms are produced via neutralization and excitation of protons due to electron transfer process from furan or THF molecules to the $\mathrm{H}^{+}$projectiles. In addition, the depopulation factors of the hydrogen excited states have been determined at each impact energy. The energetic dependences of the $K$ factors indicate that the population of higher excited states of the $\mathrm{H}(n)$ atoms depends on the selected cation. In particular, higher excited states of the $\mathrm{H}(n)$ fragments are not effectively populated in proton interaction with furan and THF molecules most probably due to the electric field which caused mixing of those states. Moreover, protons triggered a somewhat different collisional response of both target molecules - the $K$ factors are slightly higher for collisions with furan. Furthermore, the $K$ values close to -3 in the $\mathrm{C}^{+} / \mathrm{O}^{+}+\mathrm{THF}$ collisions may be taken as an indication of emission by hydrogen stemming from dissociation of the target molecule.

In conclusion, the collisions of the molecular targets with the cationic beams provide a powerful tool for all the fields, where the elucidation of physicochemical reactions and molecular manipulation is essential. In particular, the present studies may directly be applied to develop more realistic diagnostic and software packages based on the Balmer decrements that are of interest in the astrochemistry, plasma physics, medicine and the ion beam processing and fabrication.

\section{Author contribution statement}

T.J.W. conceived and designed of the study, performed the measurements, analysed and interpreted of data, created artwork, and wrote the article. B.P. performed the measurements.

This work was conducted within the framework of the COST Action CM 1204 (XLIC). B.P. acknowledges the financial support from University of Gdansk Grant UG: 530-5200-D464-14.

\section{References}

1. C Zeitlin et al., Science 340, 1080 (2013)

2. U. Feldman, E. Landi, N.A. Schwadron, J. Geophys. Res. 110, A07109.1 (2005)

3. Y.-J. Kuan, S.B. Charnley, H.-C. Huang, Z. Kisiel, P. Ehrenfreund, W.-L. Tseng, C.-H. Yan, Adv. Space Res. 33, 31 (2004)

4. S.B. Charnley, P. Ehrenfreund, Y.-J. Kuan, Spectrochim. Acta A 57, 685 (2001)

5. U. Amaldi, G. Kraft, Rep. Prog. Phys. 68, 1861 (2005)

6. I. Utke et al., J. Vac. Sci. Technol. B 26, 1197 (2008)

7. J.E.M. McGeoch, J. Microsc. 227, 172 (2007)

8. C.O. Kappe, S.S. Murphree, A. Padwa, Tetrahedron 53, 14179 (1997)

9. S. Glenis, M. Benz, E. LeGoff, J.L. Schindler, C.R. Kannewurf, M.G. Kanatzidis, J. Am. Chem. Soc. 115, 12519 (1993)

10. M. Mascal, E.B. Nikitin, Angew. Chem. Int. Ed. 47, 7924 (2008)

11. Y. Roman-Leshkov, C.J. Barrett, Z.Y. Liu, J.A. Dumesic, Nature 447, 982 (2007)

12. S. Moro, J.K. Chipman, J.-W. Wegener, C. Hamberger, W. Dekant, A. Mally, Mol. Nutr. Food Res. 56, 1197 (2012)

13. M. Anese, L. Manzocco, S. Calligaris, M.C. Nicoli, J. Agric. Food Chem. 61, 10209 (2013)

14. T.J. Wasowicz, B. Pranszke, J. Phys.: Conf. Series 635, $032055(2015)$

15. T.J. Wasowicz, B. Pranszke, J. Phys. Chem. A 119, 581 (2015)

16. Ch. Ottinger, in Gas Phase Ion Chemistry, edited by M. Bowers (Academic Press, New York, 1984), Vol. 3, p. 249

17. A. Ehbrecht, A. Kowalski, Ch. Ottinger, Chem. Phys. Lett. 284, 205 (1998)

18. T.J. Wasowicz, B. Pranszke, J. Phys. Chem. A 120, 964 (2016)

19. B.I. Moiseiwitsch, S.J. Smith, Rev. Mod. Phys. 40, 238 (1968)

20. D.W.O. Hedddle, J.W. Gallagher, Rev. Mod. Phys. 61, 221 (1989)

21. B. Van Zyl, H. Neumann, H.L. Rothwell Jr., R.C. Amme, Phys. Rev. A 21, 716 (1980)

22. B. Van Zyl, H.L. Rothwell Jr., H. Neumann, Phys. Rev. A 21, 730 (1980)

23. B. Van Zyl, M.W. Gealy, H. Neumann, Phys. Rev. A 33, 2333 (1986)

24. R. Hoekstra, F.J. de Heer, R. Morgenstern J. Phys. B 24, 4025 (1991)

25. M. Dampc, M. Zubek, Int. J. Mass Spectrom. 277, 52 (2008)

26. T.J. Wasowicz, A. Kivimäki, M. Dampc, M. Coreno, M. de Simone, M. Zubek, Phys. Rev. A 83, 033411 (2011)

27. T.J. Wasowicz, A. Kivimäki, M. Coreno, M. Zubek, J. Phys. B 45, 205103 (2012)

28. T.J. Wasowicz, A. Kivimäki, M. Coreno, M. Zubek, J. Phys. B 47, 055103 (2014)

29. I. Linert, I. Lachowicz, T.J. Wasowicz, M. Zubek, Chem. Phys. Lett. 498, 27 (2010)

30. T.J. Wasowicz, I. Linert, I. Lachowicz, M. Zubek, Photon. Lett. Pol. 3, 110 (2011)

31. J. Luque, D.R. Crosley, LIFBASE: Database and Spectral Simulation (Version 1.5). SRI International Report MP 99009, 1999 
32. J. Brzozowski, P. Bunker, N. Elander, P. Erman, Astrophys. J. 207, 414 (1976)

33. M. Zachwieja, J. Mol. Spec. 170, 285 (1995)

34. J. Luque, D.R. Crosley, J. Chem. Phys. 104, 2146 (1996)

35. J. Luque, D.R. Crosley, J. Chem. Phys. 104, 3907 (1996)

36. J. Luque, D.R. Crosley, Chem. Phys. 206, 185 (1996)

37. N. Elander, M. Hehenberger, P.R. Bunker, Phys. Scr. 20, 631 (1979)

38. M. Zubek, T.J. Wasowicz, I. Dąbkowska, A. Kivimäki, M. Coreno, J. Chem. Phys. 141, 064301 (2014)

39. B. Pranszke, Chem. Phys. Lett. 484, 24 (2009)

40. M.C. Bacchus-Montabonel, J. Phys. Chem. A 117, 14169 (2013)

41. M.C. Bacchus-Montabonel, J. Phys. Chem. A 118, 6326 (2014)

42. T.J. Wasowicz, J. Phys.: Conf. Series 635, 032114 (2015)

43. M. Zubek, M. Dampc, I. Linert, T. Neumann, J. Chem. Phys. 135, 134317 (2011)

44. M.C. Bacchus-Montabonel, M. Łabuda, Y.S. Tergiman, J.E. Sienkiewicz, Phys. Rev. A 72, 052706 (2005)

45. M. Dampc, I. Linert, M. Zubek, J. Phys. B 48, 165202 (2015)

46. A. Dominguez et al., Astrophys. J. 763, 145 (2013)

47. G. La Mura, L.C. Popovic, S. Ciroi, P. Rafanelli, D. Ilic, Astrophys. J. 671, 104 (2007)

48. W. Songbai, L. Guangjiu, L. Dongping, Y. Size, Plasma Sci. Technol. 16, 219 (2014)
49. Yu.A. Lebedev, I.L. Epstein, E.V. Yusupova, High Temp. 52, 150 (2014)

50. H.E. Bethe, E.E. Salpeter, Quantum Mechanics of One- and Two-Electron Atoms (Plenum Publishing Corporation, New York, 1977)

51. D.M. Duffy, A.M. Rutherford, J. Phys.: Condens. Matter 19, 016207 (2007)

52. L. Windholz, R. Drozdowski, T.J. Wasowicz, J. Kwela, Proc. SPIE. 5849, 24 (2005)

53. L. Windholz, R. Drozdowski, T.J. Wasowicz, J. Kwela, Opt. Appl. 36, 569 (2006)

54. L. Windholz, E. Winklhofer, R. Drozdowski, J. Kwela, T.J. Wasowicz, J. Heldt, Phys. Scr. 78, 065303 (2008)

55. L. Windholz, T.J. Wasowicz, R. Drozdowski, J. Kwela, J. Opt. Soc. Am. B 29, 934 (2012)

56. A. Mlynski, Poznan Univ. Tech. Acad. J.: Electrical Eng. 73, 147 (2013)

57. N.F. Ramsey, in Atomic, Molecular, and Optical Physics: Atoms and Molecules, edited by F.B. Dunning, R.D. Hulet (Academic Press, Inc., 1996), Vol. 29B

Open Access This is an open access article distributed under the terms of the Creative Commons Attribution License (http://creativecommons.org/licenses/by/4.0), which permits unrestricted use, distribution, and reproduction in any medium, provided the original work is properly cited. 